\title{
Riesgo de maltrato en personas adultas mayores: validación de contenido de una escala
}

\section{Older adults at risk of abuse: content validity of a scale}

\section{(1) Psicogente EISSN 2027-212X}

ARTíCULO DE
INVESTIGACIÓN
Copyright $\odot 2018$
by Psicogente

Correspondencia de autores:

dpabon1@udi.edu.co

Recibido: 17-04-17 Aceptado: $16-05-18$ Publicado: 08-01-19

\author{
Daysy Katherine Pabón-Poches \\ Universidad de Investigación y Desarrollo - UDI, Bucaramanga, Colombia
}

\section{Resumen}

Objetivo: Realizar la validación de contenido en una escala que mide el riesgo de maltrato en personas adultas mayores.

Método: Diseño instrumental, en el que se seleccionaron cuatro jueces expertos con experiencia en el área para llevar a cabo la evaluación de contenido de la escala; el proceso se realizó cuatro veces hasta conseguir el total acuerdo entre los jueces, según el método de juicio de expertos en la modalidad de agregados individuales y finalmente, se aplicó una prueba piloto.

Resultados: Los jueces expertos Ilegaron a un total acuerdo respecto a la claridad, coherencia, lenguaje, relevancia de los ítems y suficiencia de las categorías planteadas tras cuatro revisiones, permitiendo la configuración de la escala con tres categorías y 80 ítems. Con la aplicación piloto se encontró que 66,7 \% de los mayores reportaron maltrato, el tipo de maltrato más frecuente fue el psicológico (29,2 \%), se evidencian pocos factores de riesgo, aunque se destaca la presencia de estereotipos negativos sobre la vejez por parte de los mismos gerontes y limitaciones económicas.

Conclusión: Con la validación de contenido la escala logra evaluar los factores de riesgo de maltrato del adulto mayor con claridad en la redacción, coherencia interna en cada ítem, sin inducción a las respuestas, un lenguaje adecuado a la población, dejando solo los ítems relevantes y permitiendo que estos fueran suficientes para la evaluación de las tres dimensiones propuestas; la aplicación de la prueba piloto permitió corroborar dicha validez.

Palabras clave: Maltrato, Adulto mayor, Riesgo de maltrato, Validación de contenido.

Abstract

Objective: To perform content validation on a risk scale that measures the risk of abuse in older adults.

Method: Instrumental design, in which four expert judges with experience in the area were selected to carry out the content evaluation of the scale; the process was realized four times until full agreement was reached among the judges, according to the expert judgment method in the individual aggregate modality and finally, a pilot test was applied.

Results: The expert judges reached a total agreement regarding the clarity, coherence, language, relevance of the items and sufficiency of the proposed categories after four revisions, allowing the configuration of the scale with three categories and 80 items. With the pilot application it was found that $66.7 \%$ of the elders reported abuse, the most frequent type of abuse was the psychological one (29.2\%), there are few risk factors, although the presence of negative stereotypes about the old age on the part of the same older adults and economic limitations

Conclusion: With the validation of content the scale manages to assess the risk factors of abuse of the elderly with clarity in the writing, internal coherence in each item, without induction to the answers, a language appropriate to the population, leaving only the relevant items and allowing these to be sufficient for the evaluation of the three proposed dimensions; the application of the pilot test allowed corroborating this validity.

Key words: Abuse, Elder abuse, Risk of abuse, Content validity.

Cómo citar este artículo (APA):

Pabón-Poches, D. K. (2019). Riesgo de maltrato en personas adultas mayores: validación de contenido de una escala. Psicogente 22(41), 1-27. https://doi. org/10.17081/psico.22.41.3299 


\section{INTRODUCCIÓN}

En el mundo se ha evidenciado una reducción de la natalidad, fecundidad, mortalidad, y un incremento en la esperanza de vida al nacer, lo que ha generado que en la actualidad se esté experimentando un proceso de envejecimiento poblacional. En Colombia, se estima que para el 2020 la esperanza de vida al nacer será de 73,08 años para los hombres y 79,39 años para las mujeres (Departamento Administrativo Nacional de Estadística, DANE, 2009), en Santander será de 79 años para las mujeres y 73 años para los hombres, proyectando un incremento de la población adulta mayor de 14 \% (LoaizaValencia, 2015); en la actualidad estos datos representan un logro, pero también un desafío ya que los prejuicios y los estereotipos suelen desencadenar situaciones de discriminación y desigualdad, además de reforzar la connotación negativa que ha adquirido la vejez y el envejecimiento (Fernández, 2015; Rodríguez, 2011).

El proceso de envejecimiento trae consigo cambios individuales configurados por ganancias y pérdidas; sin embargo, la imagen negativa sobre la vejez y discriminación por la edad, actitudes edadistas, la violencia intergeneracional y la cultura violenta que predomina en la sociedad, hacen más evidente la pérdida que la ganancia y facilitan la aparición de fenómenos como el maltrato (Moreno \& González, 2010; Tabueña, 2009).

El maltrato es comprendido como la acción que ocasiona daño físico o psicológico a una persona anciana de forma única o repetida y/o por falta de acción oportuna, que se genera dentro de cualquier relación donde exista una expectativa de confianza (Huenchuan, 2012; Tabueña, 2009), y del cual se pueden distinguir cinco tipos, el maltrato físico, el psicológico o emocional, económico o explotación material, negligencia y abuso sexual (Giraldo, 2012).

Aunado a los tipos de maltrato, se encuentran los factores de riesgo, los cuales son considerados como características personales, familiares, escolares, laborales, sociales o culturales cuya presencia hace que aumente la probabilidad de que se produzca un fenómeno determinado, en este caso concreto refieren a variables que ubican a los mayores en una posición vulnerable hacia las conductas y actitudes violentas (Iborra, 2008).

Pabón y Delgado (2017) a través de una revisión bibliográfica describen la clasificación de tres factores de riesgo hallados en la literatura sobre maltrato, destacando los referidos a la persona mayor, los asociados al cuidador y los relacionados con aspectos sociales, lo que incluye el contexto social, las 
instituciones, el entorno y la cultura. Algunos factores de riesgo asociados al mayor son la presencia de deterioro físico, cognitivo y alteraciones en la conducta, depresión, baja autoestima, y dependencia emocional o financiera (Bover, Moreno, Mota \& Taltavull, 2003; Iborra, 2008; Moreno \& González, 2010); en cuanto a los factores de riesgo asociados al cuidador se destaca la presencia de sobrecarga física y emocional, depresión, abuso de alcohol, fármacos o droga, sentimientos de frustración, baja autoestima, soledad, ansiedad y enfermedad física crónica, entre otros (Bover et al., 2003; Iborra, 2008; Moreno \& González, 2010; Tabueña, 2009); como factores de riesgo sociales, están los asociados a las instituciones o al contexto socioestructural, como centros de salud; se encuentran los servicios de mala calidad, personal poco preparado, que recibe bajos salarios y con sobrecarga; riesgo sociocultural o asociado al entorno, como aislamiento social, edadismo, cultura violenta y pérdida de la estabilidad económica, entre otros (Bover et al., 2003; Iborra, 2008; Moreno \& González, 2010; Tabueña, 2009).

Dentro de los hallazgos del estudio del maltrato se encuentra el aporte de Eslami, et al. (2016), quienes hallaron en una muestra de 4.467 adultos mayores de siete países europeos que más del $34 \%$ de los participantes informaron experimentar una vida de maltrato psicológico, 11,5\% físico, $18,5 \%$ financiero y $5 \%$ de abuso sexual y $4,3 \%$ reportaron lesiones. Por otro lado, Ruelas-González et al. (2016) con 8.894 personas mayores de 60 años de México, identificaron una prevalencia de maltrato de $1,7 \%$, donde predominaron las agresiones verbales $(60 \%)$ dentro del hogar $(37,6 \%)$ en las mujeres y agresiones físicas, en la vía pública en el caso de los hombres $(30,7 \%)$, donde mayormente, los agresores no tenían un vínculo filial con los gerontes afectados.

Respecto al estudio del maltrato en Colombia, Pabón y Delgado (2017) resaltan un reporte escaso, donde hay evidencia de publicaciones de tipo descriptivo sobre demandas en Comisarías de Familia o encuestas generales aplicadas a mayores que han referido el maltrato psicológico, como el más frecuente entre las denuncias (Cardona-Arango et al., 2010; García \& Medina, 2009; Sepúlveda et al., 2009). Así mismo, se destaca el estudio de Cano, Garzón, Segura \& Cardona (2015), quienes realizaron un estudio transversal descriptivo, de enfoque empírico-analítico donde identificaron como variables explicativas del maltrato el estrato socioeconómico, tipo de vivienda, etnia, riesgo de depresión, riesgo de ansiedad, riesgo de deterioro cognitivo y red de apoyo escasa. 
Basados en lo anterior, se identifican dos focos importantes para el abordaje del tema, en primera medida, el escaso reporte de estudios desde el área de psicología (Pabón \& Delgado, 2017), a pesar de ser este el tipo de maltrato con mayor mención en los hallazgos de estudios realizados en Colombia y de constituir una problemática de salud pública que ha ido en incremento, y que demanda visibilidad y atención (Barrón, Sánchez \& Méndez, 2002); en segundo lugar, la evaluación de este fenómeno por medio de encuestas poco claras, sin mención sobre procesos de validación, haciendo visibles limitaciones de validez y de los alcances de los resultados aportados por medio de los instrumentos utilizados.

Este trabajo plantea como objeto de estudio realizar la validación de contenido de una escala de riesgo de maltrato en personas adultas mayores, la cual permita identificar factores de riesgo de maltrato asociados al adulto mayor, factores de riesgo asociados a su cuidador y factores de riesgo asociados al entorno sociocultural; por lo anterior, este estudio se cuestiona sobre ¿cómo validar una escala de factores de riesgo de maltrato en personas adultas mayores?

Según Mora-Ríos, Bautista-Aguilar, Natera y Pedersen (2013) no existe un consenso sobre la forma más adecuada para adaptar instrumentos, existen diversos enfoques y estrategias donde basados en los objetivos, alcances y recursos el investigador debe seleccionar el más apto, pero sin duda, la validación de un instrumento permitirá rescatar propiedades de validez y confiabilidad necesarias para su aplicación.

La validez de un instrumento consiste en la autenticidad, en que mida lo que pretende medir y existen diferentes tipos de validez: de contenido, de constructo y validez predictiva, concurrente o de elaboración de hipótesis (Aragón, 2011; Corral, 2009; Pedrosa, Suárez-Álvarez \& García-Cueto, 2013). Sin embargo, en este estudio el interés recae sobre la validez de contenido que refiere al grado en que un instrumento refleja un dominio específico del contenido que se quiere medir, determinando la capacidad del ítem o reactivo para capturar la/las dimensión(es) que la prueba pretende medir (Corral, 2009; Escobar-Pérez \& Cuervo-Martínez, 2008).

Adicionalmente, Urrutia, Barrios, Gutiérrez y Mayorga (2014) plantean que una validación de contenido puede utilizar como métodos los resultados de la prueba, opinión de evaluados, entrevista cognitiva y evaluación por parte del experto; por otro lado, también se emplean fórmulas factoriales 
con ecuaciones estructurales, las cuales son menos comunes. Así mismo, se parte de la idea de que desarrollar un proceso de adaptación que logre que el instrumento sea culturalmente apropiado, debe tener en cuenta cuatro criterios fundamentales según Manson (1997, citado por Mora-Ríos et al., 2013): 1) nivel de comprensión, 2) aceptabilidad, 3) relevancia, e 4) integridad semántica/conceptual.

La manera más común y eficaz para llevar a cabo la validación de contenido de un instrumento ha sido por panel o juicio de expertos (Urrutia et al., 2014), este es definido como "la opinión informada de personas con trayectoria en el tema, que son reconocidos por otros como expertos cualificados que pueden dar información, evidencia, juicios y valoraciones" (Escobar-Pérez \& CuervoMartínez, 2008). Mediante el juicio de expertos, se logran obtener estimaciones sobre la probabilidad de error en la configuración del instrumento; este método al mismo tiempo, se puede dar grupal, por experto único o en la modalidad de agregados individuales, método Delphi, técnica de grupo nominal y método de consenso grupal (Corral, 2009).

El método a usarse en este estudio, es el de agregados individuales. Este consiste en solicitar a cada experto una estimación directa de los ítems del instrumento; no exige la reunión de los jueces, es económico y se evita el sesgo de los datos ya que se elude la presión entre expertos o conflictos interpersonales (Corral, 2009; García-Valdés \& Suárez-Marín, 2013).

Un instrumento sobre maltrato es propuesto por Touza, Prado y Segura (2011), quienes llevan a cabo un estudio que pretendía crear y validar un instrumento de detección de situaciones de riesgo de malos tratos domésticos y comportamientos autonegligentes hacia las personas mayores, para ser utilizado por los profesionales de los servicios sociales. Resaltando índices de consistencia interna elevados y una adecuada estabilidad temporal en sus puntuaciones, aplicados a población española.

Por otro lado, Giraldo-Rodríguez (2006), quien diseña y aplica la Encuesta sobre maltrato a personas adultas mayores en el Distrito Federal, México, con el objetivo de describir la percepción del maltrato en las personas adultas mayores, los tipos de maltrato y otros factores asociados, refleja un análisis estadístico sobre los resultados obtenidos en los sujetos evaluados.

Teniendo en cuenta la relevancia del maltrato en la población mayor, el reporte existente sobre el tema en Colombia, la metodología utilizada en 
las validaciones de contenido y otros criterios adicionales; este estudio se plantea trabajar sobre la validación de contenido de una escala de riesgo de maltrato que permita una evaluación más fiable de este fenómeno.

\section{MÉTODO}

\subsection{Diseño}

Estudio de tipo instrumental (Ato, López \& Benavente, 2013), ya que se orienta al desarrollo de una escala, específicamente la validación de contenido por medio del método de juicio de expertos en la modalidad de agregados individuales; dicha modalidad consiste en solicitar a cada experto una valoración cuantitativa y una apreciación cualitativa sobre los ítems del instrumento a validar (Corral, 2009).

\subsection{Participantes}

Cuatro jueces expertos seleccionados por conveniencia, cumplimiento como criterio de selección, tener una formación académica o investigativa relacionada con el abordaje de la población adulta mayor, mínimo 10 años de experiencia en el ejercicio profesional o investigativo. De esta manera, se seleccionó un doctor en psicología con más de diez años de experiencia en el trabajo clínico e investigativo con la población mayor, un gerontólogo con más de 25 años de experiencia en el trabajo con gerontes, un magíster con experiencia en el diseño y validación de pruebas y un magíster docente investigador de psicología.

Para la prueba piloto se contó con la participación de 24 adultos mayores, 17 mujeres y 7 hombres pertenecientes a un grupo de autoayuda y centro vida de una institución de Floridablanca, entre 65 y 80 años de edad, con un promedio de 71,83 años, en su mayoría casados $(45,8 \%)$ con primaria incompleta $(37,5 \%)$ y completa $(37,5 \%)$, pertenecientes a los estratos $2(45,8 \%)$ y $3(29,2 \%)$ participantes en la aplicación de la prueba piloto.

Estos cumplían como criterios de inclusión: no estar institucionalizados, lograr mantener una conversación fluida, permanecer conscientes y ajustados a la realidad. 


\subsection{Instrumentos}

\subsubsection{Ficha de evaluación por parte del experto}

Formato adaptado de Corral (2009) y Escobar-Pérez y Cuervo-Martínez (2008). Consta de dos secciones, la primera, corresponde a la aceptación de la participación como juez en el estudio y la segunda, la rúbrica con la que evaluará los ítems de la escala, dicha evaluación incluye como criterios: la claridad en la redacción, la coherencia interna, la inducción a la respuesta (sesgo), el uso de lenguaje adecuado con el nivel del informante y si mide lo que pretende medir (validez). Ante cada uno de estos criterios el juez responde "SÍ" o "NO", según el caso y si considera necesario realizar aclaraciones o comentarios, cuenta con un espacio adicional para observaciones por ítem, el cual se sugiere utilizar en caso de que la respuesta indique una falla o debilidad en el ítem.

Finalmente, la segunda sección solicita la evaluación de tres aspectos generales de la escala, que podrá evaluar marcando con una equis la opción "Sí" o "NO" y en definitiva, valorar la escala como: aplicable, no aplicable o aplicable atendiendo a las observaciones y modificaciones sugeridas (Anexo 1).

\subsubsection{Escala geriátrica de maltrato al adulto mayor}

Validada por Giraldo-Rodríguez y Rosas-Carrasco (2013). Está formulada para adultos mayores de 60 años no institucionalizados, presenta una duración entre 8 y 20 minutos (dependiendo de las características del adulto mayor) y su forma de aplicación es administrada de forma individual. Su objetivo principal es el de obtener información sobre la dimensión, caracterización y prevalencia del maltrato hacia personas adultas mayores.

Está compuesta por 22 ítems que exploran maltrato físico, psicológico, negligencia, maltrato económico y sexual; adicionalmente, permite indagar por la frecuencia, duración, responsable de la agresión y género. Se considera como presencia de maltrato o presencia de un tipo de maltrato específico ante una afirmación positiva en uno de los 22 ítems.

\subsubsection{Encuesta sobre maltrato a personas adultas mayores en el Distrito} Federal EMPAM- DF adaptada de Giraldo-Rodríguez (2006)

Consta de 91 ítems distribuidos en seis secciones, y dos iniciales de datos de identificación y datos personales: 


\section{Datos de identificación}

\section{II. / III. Características de la vivienda y residentes del hogar}

IV. Información laboral y de ingresos: proporciona datos sobre la situación laboral, posición en el trabajo, nivel y fuentes de ingresos de la persona adulta mayor.

V. Factores de riesgo: realiza una valoración integral y un diagnóstico de problemas que presenta la persona adulta mayor y su entorno, permitiendo destacar los posibles factores de riesgo asociados al maltrato. Incluye: Valoración física (describe su grado de dependencia y estado general de salud), valoración psicológica (describe aspectos como la soledad, la comunicación, la autonomía, el deterioro cognitivo, el estado de ánimo y la autopercepción), valoración de signos financieros (describe la participación de la persona adulta mayor en asuntos económicos).

VI. Información de la(s) persona(s) que le cuida(n) o ayuda(n): permite conocer quién es la persona que le cuida, sexo, edad, si recibe remuneración, conocer la relación económica existente con el adulto mayor, tiempo de cuidado y la percepción del adulto mayor respecto al tipo de relación que llevan.

VII. Negligencia institucional: busca conocer la problemática que enfrenta la persona mayor cuando acude a una institución para recibir cualquier tipo de servicio e identificar si ha sido maltratada por alguna institución pública o privada.

VIII. Características del maltrato: se compone de cuatro apartados que buscan conocer la prevalencia y severidad de los malos tratos hacia los adultos mayores en los últimos doce meses. Así, se divide en: Maltrato físico, Maltrato psicológico, Negligencia y abandono, Explotación económica, Maltrato sexual.

\subsection{Procedimiento}

En la fase inicial del proyecto se realizó una revisión literaria respecto a definición, clasificación y factores de riesgo del maltrato; así mismo, se indagó sobre los instrumentos de evaluación en el contexto colombiano y latinoamericano. Ante la ausencia de instrumentos validados en este contexto y sobre la revisión de los más usados o adaptados en contextos cercanos, se decide diseñar y validar por jueces una escala que permita identificar los diferentes factores de riesgo del maltrato. 
Se define como objetivo del juicio de expertos: brindar un juicio sobre la validez de contenido de la escala, afirmando si es apto para aplicación o no, teniendo en cuenta criterios como la claridad en la redacción, la coherencia interna, la inducción a la respuesta (sesgo), el uso de lenguaje adecuado con el nivel del informante y la suficiencia de cada subcategoría. Así, se estructuró un protocolo de presentación para que los jueces accedan a toda la información de la prueba y el estudio, un instructivo para diligenciar y entregar el formato de evaluación, y finalmente, la "Ficha de evaluación por parte del experto" que incluye la firma de aceptación de cada juez.

Seguidamente, se dio paso a la selección y contacto con los expertos, estos fueron seleccionados por conveniencia, teniendo en cuenta su experiencia en evaluación de instrumentos, en investigación, por su experticia, por la existencia de publicaciones relacionadas con el tema y por ser docentes investigadores de psicología. Cuatro jueces expertos en total. Inicialmente se contactan por medio telefónico y tras aceptar la participación se enviaron todos los formatos vía correo electrónico, contando con quince días para proporcionar respuesta. Una vez realizada la evaluación, se programó una reunión con cada uno de ellos y de forma individual para recibir una realimentación verbal y los formatos diligenciados. Este proceso se llevó a cabo tres veces, hasta que se logró un total acuerdo en la evaluación realizada por los jueces; en cada evaluación participaron tres expertos, en la segunda y la tercera se generó un cambio de juez.

Ante cada recepción de los formatos de evaluación se tabularon las respuestas de cada juez en Excel, donde "uno" indicaba la presencia del indicador y "dos" su ausencia (el único indicador que debía estar ausente era sesgo). En el juicio de expertos se eliminaron ítems ante el total acuerdo de los jueces y así mismo se dejaron intactos los que contaban con toda la aprobación de los expertos, aquellos que presentaban $67 \%$ del acuerdo, que corresponde a dos jueces de acuerdo tenían modificaciones, según el indicador en el que fallaran.

Con los datos tabulados, se procedió a modificar la escala, en la que las dimensiones que la componen se reestructuraron y se obtuvo un incremento en el número de ítems de 91 a 121, mejorando la relevancia de los ítems incluidos, la coherencia interna, reduciendo el sesgo e incrementando la suficiencia para la escala final. En la segunda evaluación se redujeron a 80 ítems y en la tercera evaluación se obtuvo el acuerdo total entre los pares expertos. 
Una vez se estableció la escala final, se procedió a llevar a cabo la aplicación de la prueba piloto con 24 adultos mayores no institucionalizados pertenecientes al centro vida y a diferentes grupos de autoayuda de la ciudad de Floridablanca, quienes autorizaron a la investigadora para solicitar la participación de los mayores vinculados y realizar las aplicaciones dentro de las instalaciones; así, se pactó cita con 24 mayores que aceptaron participar voluntariamente y quienes dieron respuesta a los formatos, incluido consentimiento informado, Escala Geriátrica de Maltrato al Adulto Mayor (Giraldo-Rodríguez \& Rosas-Carrasco, 2013) para identificar su presencia y la Escala de Riesgo de Maltrato en Personas Adultas Mayores Adaptada en su versión final, todo ello dentro de las instalaciones de la entidad. Posterior a la aplicación se da paso a la tabulación y análisis de datos, que conforman un informe final de resultados.

\subsection{Análisis de datos}

Se realiza a través del programa SPSS, por medio de estadísticos descriptivos que indican frecuencias y porcentajes de la presencia de maltrato, los tipos de maltrato predominantes y los factores que representan riesgo.

\subsection{Conflicto de interés}

No existe conflicto de intereses. El trabajo es realizado por una sola autora, apoyada por la Universidad de Investigación y Desarrollo.

\section{RESULTADOS}

Los resultados de la primera evaluación de la escala de maltrato adaptada de Giraldo-Rodríguez (2006) permitió dar cuenta de varios aspectos: la forma de enunciar los datos sociodemográficos debía cambiar, esto afectó la relevancia de las dos primeras categorías; el tipo de respuesta se debía unificar para evitar afectar la claridad y coherencia especialmente por las opciones de respuesta, presentar los ítems como afirmaciones y no como preguntas, estos aspectos afectaron la claridad y relevancia, se debían modificar los nombres de las categorías valoración física, valoración psicológica, valoración de signos financieros, adicionalmente, en las categorías abuso sexual, valoración psicológica, información de las personas que cuidan, negligencia institucional y valoración física no se consideraron suficientes y se recomendó agregar ítems; se identificaron modificaciones puntuales en cuanto a redacción y reubicación de categoría en 16 ítems de diferentes categorías, y se sugirió eliminar siete reactivos. 
Por lo anterior, se modificaron los ítems señalados, las dimensiones y subdimensiones, atendiendo a los comentarios y enunciando una nueva escala, con un objetivo definido: evaluar los factores de riesgo de maltrato en población mayor.

Tabla 1.

Resultados segunda evaluación de jueces expertos sobre el contenido de la escala

\begin{tabular}{|c|c|c|c|c|}
\hline \multirow[b]{2}{*}{ DIMENSIÓN } & \multirow[b]{2}{*}{ INSUFICIENTE } & \multirow[b]{2}{*}{ SUBDIMENSIÓN } & \multicolumn{2}{|c|}{ CAMBIOS } \\
\hline & & & $\begin{array}{l}\text { No ÍTEMS A } \\
\text { ELIMINAR }\end{array}$ & $\begin{array}{l}\mathrm{N}^{0} \text { ÍTEMS A } \\
\text { MODIFICAR }\end{array}$ \\
\hline \multirow{5}{*}{$\begin{array}{l}\text { Factores de Riesgo Adulto } \\
\text { Mayor (53 ítems) }\end{array}$} & \multirow{4}{*}{ sí } & Físicos (13 ítems) & 5 & \\
\hline & & Emocionales (14 ítems) & 2 & 2 \\
\hline & & Cognitivos (8 ítems) & 1 & 1 \\
\hline & & Económicos (11 ítems) & 2 & \\
\hline & \multirow{3}{*}{ sí } & Autonegligencia (7 ítems) & 1 & 7 \\
\hline \multirow{2}{*}{$\begin{array}{l}\text { Factores de Riesgo Asociados } \\
\text { al Cuidador (30 ítems) }\end{array}$} & & Características (11 ítems) & 1 & \\
\hline & & Factores de Riesgo (19 ítems) & 2 & \\
\hline \multirow{3}{*}{$\begin{array}{l}\text { Factores de Riesgo Asociados } \\
\text { al Entorno Cultural y las } \\
\text { instituciones (38 ítems) }\end{array}$} & \multirow{3}{*}{ sí } & Asociados al Entorno (18 ítems) & 13 & \\
\hline & & Asociados a la Cultura (13 ítems) & 6 & 1 \\
\hline & & Asociados a las Instituciones ( 7 ítems) & 8 & \\
\hline
\end{tabular}

La Tabla 1 refleja los resultados de la segunda evaluación por jueces; la escala entregada estaba conformada por 121 ítems, distribuidos en tres grandes dimensiones, tras la evaluación se determinó por total acuerdo, eliminar 41 ítems, y modificar 11 ítems por carencia en la claridad, coherencia interna y lenguaje poco acorde a la población. La mayoría de ítems eliminados se enmarcaron en las categorías asociados al entorno, a la cultura y las instituciones, en general se consideraron repetitivos y se sugirió además unirlos en una sola dimensión.

Tabla 2.

Resultados tercera evaluación de jueces expertos sobre el contenido de la escala

\begin{tabular}{cc}
\hline DISTRIBUCIÓN DIMENSIONES & SUBDIMENSIÓN \\
\hline Factores de Riesgo Adulto Mayor (45 ítems) & Físicos ( 8 ítems) \\
& Emocionales (13 ítems) \\
& Cognitivos ( 7 ítems) \\
Factores de Riesgo Asociados al Cuidador & Económicos (11 ítems) \\
Factores de Riesgo Asociados al Entorno, Cultural y las instituciones & Auto-negligencia ( 6 ítems) \\
\hline
\end{tabular}


La Tabla 2 refleja la organización enviada en la tercera evaluación y la que recibió una aprobación total por los tres jueces, conformando así la versión aprobada para aplicación (Anexo 2).

De la aplicación de la prueba piloto de la escala, la cual tenía como propósito caracterizar el riesgo de maltrato de los mayores evaluados, se evidenció que $66,7 \%$ de los mayores reportan maltrato, el tipo más frecuente entre los 16 gerontes con presencia del mismo fue el psicológico $(29,2 \%)$, por otro lado, 29,2 \% reportó sufrir más de un tipo de maltrato, entre ellos el psicológico, el físico y la negligencia, por último 2 de los 16 mayores reportaron maltrato de tipo sexual. En cuanto al parentesco del agresor, generalmente fue el esposo(a) (25\%) o nuero(a) (8,3\%), mientras que el $16,7 \%$ indicó que el maltrato se dio por varios familiares.

Tres de los evaluados reportaron no tener cuidador y del 87,5 \% que reportó tener cuidador, el $50 \%$ indicó que el parentesco de la persona de apoyo era hijo(a), 66,7 \% señala presentar una enfermedad crónica y 91,7 \% reporta ausencia de algún diagnóstico psiquiátrico, ninguno presentó desnutrición, pero sí sobrepeso $(37,5 \%)$ y obesidad tipo I (16,7 \%).

Tabla 3.

Factores de riesgo físicos del adulto mayor

\begin{tabular}{ccc}
\hline ÍTEM & \multicolumn{2}{c}{ Sí } \\
\cline { 2 - 3 } Presenta alteraciones visuales & $\%$ & N \\
\hline Pas alteraciones visuales le dificultan realizar las actividades diarias & 83,3 & 20 \\
Presenta alteraciones auditivas & 33,3 & 8 \\
Las alteraciones auditivas le dificultan realizar las actividades diarias & 20,8 & 5 \\
Está diagnosticado con alguna patología crónica & 0 & 0 \\
La(s) enfermedades crónicas le generan dependencia & 29,2 & 7 \\
Sufre de dolores físicos intensos & 0 & 0 \\
Usted percibe su salud como deficiente & 54,2 & 13 \\
\hline
\end{tabular}

La Tabla 3 presenta el porcentaje y la frecuencia de los ítems que fueron respondidos afirmativamente. Así, del riesgo físico se resalta la presencia de alteraciones físicas y dolores intensos. 
Tabla 4.

Factores de riesgo emocionales del adulto mayor

\begin{tabular}{ccc}
\hline ÍTEM & sí \\
\cline { 2 - 3 } Siente que tiene una persona de confianza a la que le puede comunicar sus problemas & \% & N \\
Ha sentido pérdida de interés por las cosas que generalmente le gusta hacer & 87,5 & 21 \\
Se siente asustado(a) o con miedo & 33,3 & 8 \\
Llora con facilidad & 20,8 & 5 \\
Se siente a menudo aburrido & 45,8 & 11 \\
Se irrita con facilidad & 41,7 & 10 \\
Últimamente se ha sentido deprimido(a) & 41,7 & 10 \\
Últimamente se ha sentido solo(a) & 37,5 & 9 \\
Siente miedo de alguien & 37,5 & 9 \\
Tiene miedo de que le suceda algo malo & 12,5 & 3 \\
Se siente desconfiado o tímido & 41,7 & 10 \\
Se siente mal al tratar de relacionarse con los demás & 33,3 & 8 \\
Ha sentido deseos de irse a otro lugar porque se siente mal en donde vive y con quien vive & 37,5 & 9 \\
\hline
\end{tabular}

\section{Por su lado, la Tabla 4 destaca que los mayores sienten que tienen una persona de confianza a la que le pueden comunicar sus problemas; sin embargo, algunos lloran con facilidad, se sienten a menudo aburridos, irritables y con miedo de que les suceda algo.}

Tabla 5.

Factores de riesgo cognitivo del adulto mayor

\begin{tabular}{ccc}
\hline \multicolumn{1}{c}{ ÍTEM } & \multicolumn{1}{c}{ Sí } \\
\cline { 2 - 3 } Ha presentado olvidos frecuentes & $\%$ & $\mathbf{N}$ \\
Ha presentado problemas por los olvidos que presenta & 75 & 18 \\
Se desorienta con facilidad perdiendo noción del tiempo (fecha/hora) & 16,7 & 4 \\
Se desorienta con facilidad perdiendo la ubicación del lugar en el que se encuentra & 33,3 & 8 \\
Se le dificulta concentrarse & 20,8 & 5 \\
Sufre de alteraciones del sueño & 50 & 12 \\
Ha sufrido pérdida de la conciencia por caída o golpe en la cabeza & 50 & 12 \\
\hline
\end{tabular}


Respecto a los factores cognitivos, la Tabla 5 refleja que los gerontes reportan presentar olvidos frecuentes, dificultad para concentrarse y alteraciones del sueño.

Tabla 6.

Factores de riesgo económico

\begin{tabular}{ccc}
\hline ÍTEM & \multicolumn{2}{c}{ Sí } \\
\cline { 2 - 3 } Tiene ingresos mensuales propios & $\%$ & $\mathbf{N}$ \\
\hline Sus ingresos son superiores al mínimo legal vigente $(\$ 678.400)$ & 58,3 & 14 \\
Depende económicamente de su familia & 4,2 & 1 \\
Recibe pensión & 50 & 12 \\
Está jubilado & 0 & 0 \\
Actualmente trabaja & 5 & 20,8 \\
Trabaja, pero no es remunerado & 4 & 16,7 \\
Actualmente se dedica a quehaceres dentro del hogar & 1 & 4,2 \\
Necesita de la ayuda de alguien para manejar su dinero & 87,5 & 21 \\
Puede disponer de su dinero y hacer gastos con libertad & 0 & 0 \\
Delega en otra persona el manejo de su dinero & 18 & 75 \\
\hline
\end{tabular}

En la descripción de la Tabla 6 correspondiente a los factores económicos se halló que 58,3\% reporta tener ingresos mensuales propios, solo 1 de 14 supera el mínimo legal vigente $(4,2 \%)$, el $50 \%$ de ellos dependen económicamente de la familia y $87,5 \%$ se dedica a los quehaceres dentro del hogar.

Tabla 7.

Factores de riesgo por autonegligencia

\begin{tabular}{ccc}
\hline ÍTEM & \multicolumn{2}{c}{ sí } \\
\cline { 2 - 3 } Descuida su cuidado personal & $\%$ & $\mathbf{N}$ \\
Prefiere no pedir ayuda aun si la necesita & 8,3 & 2 \\
Incumple las instrucciones o recomendaciones médicas & 50 & 12 \\
Prefiere no acudir al médico aun cuando lo requiere & 25 & 6 \\
No asiste a los controles médicos programados & 16,7 & 4 \\
Prefiere estar callado(a) y no molestar a nadie & 0 & 0 \\
\hline
\end{tabular}

Respecto a la autonegligencia, $50 \%$ prefiere no pedir ayuda aun cuando la necesita y $75 \%$ de los mayores prefieren estar callados(as) y no molestar a nadie. 
Tabla 8.

Factores de riesgo asociados al cuidador

\begin{tabular}{ccc}
\hline ÍTEM & \multicolumn{1}{c}{ Sí } \\
\cline { 2 - 3 } Cuenta con una persona de apoyo que lo cuida cuando lo requiere & \% & N \\
La persona que lo apoya en sus actividades pertenece a su familia & 90,5 & 19 \\
La persona que lo apoya se dedica exclusivamente a ello & 90,5 & 19 \\
La persona que lo apoya le dedica tiempo a otras funciones & 4,8 & 1 \\
La persona que lo cuida recibe pago por ello & 57,1 & 12 \\
Mantiene una comunicación positiva con la persona que lo apoya & 4,8 & 1 \\
Percibe una relación positiva con la persona que lo apoya en sus actividades diarias & 85 & 17 \\
La persona que lo apoya depende económicamente de usted & 80 & 16 \\
La persona de apoyo se adapta a las condiciones para poder darle cuidados & 10 & 2 \\
La persona que apoya su cuidado consume alcohol con frecuencia & 70 & 14 \\
La persona que lo apoya se altera con facilidad & 0 & 0 \\
La persona que lo apoya consume medicamentos psiquiátricos & 30 & 6 \\
\hline
\end{tabular}

Del total de mayores (24) solo 85,5 \% afirmó tener una persona de apoyo. Así, de los factores de riesgo asociados al cuidador presentados en la Tabla 8 destacan que en el 90,5 \% de los casos la persona de apoyo pertenece a la familia, $100 \%$ de las personas de apoyo no se dedican exclusivamente a ello, el $57,1 \%$ de los mayores indicaron que la persona que lo apoya dedica tiempo a otras funciones, el $100 \%$ los cuidadores no reciben pago por ello, solo en el $10 \%$ de los casos la persona de apoyo depende económicamente del mayor, en el $30 \%$ el mayor percibe que el cuidador se altera con facilidad.

Tabla 9.

Factores de riesgo asociados al entorno, la cultura y las instituciones

\begin{tabular}{|c|c|c|}
\hline \multirow{2}{*}{ ÍTEM } & \multicolumn{2}{|c|}{ sí } \\
\hline & $\%$ & $\mathbf{N}$ \\
\hline La casa en donde vive es de su propiedad & 47,8 & 11 \\
\hline Usted paga arriendo & 13 & 3 \\
\hline Usted tiene su propia habitación & 100 & 24 \\
\hline Cuenta con servicios públicos básicos & 100 & 24 \\
\hline Su lugar de vivienda es aislado & 4.3 & 1 \\
\hline Tiene acceso a servicios de salud & 95,7 & 22 \\
\hline La zona en donde vive es insegura & 4.3 & 1 \\
\hline Se ha visto afectado por la inseguridad de la zona en donde vive & 0 & 0 \\
\hline $\begin{array}{c}\text { Ha recibido algún tipo de maltrato en instituciones, empresas, entidades, locales por ser usted } \\
\text { una persona mayor }\end{array}$ & 4,3 & 1 \\
\hline Se ha sentido maltratado por algún profesional de la salud & 4.3 & 1 \\
\hline Se ha sentido atendido por personal poco capacitado & 26,1 & 6 \\
\hline Tiene una relación cercana con sus hijos & 60,9 & 14 \\
\hline Tiene una relación cercana con sus nietos & 52,2 & 12 \\
\hline
\end{tabular}




\begin{tabular}{ccc}
\hline ÍTEM & \multicolumn{2}{c}{ Sí } \\
\cline { 2 - 3 } & \% & N \\
\hline Siente que en su familia piensan que por ser mayor es una carga & 34,8 & 8 \\
Usted piensa que por ser mayor es una carga & 39,1 & 9 \\
Piensa que la vejez presenta solo pérdidas & 52,2 & 12 \\
En su familia hablan de forma negativa de las personas mayores & 8,7 & 2 \\
En su familia han existido casos de maltrato & 8,7 & 2 \\
Alguna vez se ha denunciado un caso de maltrato en su familia & 13 & 3 \\
En su barrio conoce casos de maltrato & 17,4 & 4 \\
En su infancia sufrió maltrato por parte de sus padres & 47,8 & 11 \\
En su matrimonio sufrió maltrato por parte de su pareja & 30,4 & 7 \\
Siente que le ofrecen servicios deficientes por ser mayor & 8,7 & 2 \\
\hline
\end{tabular}

Finalmente, la Tabla 9 evidencia los factores de riesgo asociados al entorno, la cultura y las instituciones; se encontró que $13 \%$ de los mayores paga arriendo, 52,2 \% refiere que la vejez representa solo pérdidas, 47,8 \% afirma que durante su infancia sufrió maltrato por parte de sus padres.

\section{DISCUSIÓN}

La implementación del método de panel o juicio de expertos en la modalidad de agregados individuales permitió llevar a cabo la validación de contenido de la escala sobre riesgo de maltrato en personas mayores, pues además de ser un método ampliamente utilizado para validaciones de contenido, muestra efectividad en el proceso de validación (Corral, 2009; Escobar-Pérez \& Cuervo-Martínez, 2008; Pedrosa et al., 2013; Urrutia et al., 2014), así mismo, presenta ventajas que incluyen favorecer la libertad de opiniones, reducir influencias dadas en interacciones de grupo, confidencialidad de las respuestas dadas por los expertos y reúne y sintetiza el conocimiento de un grupo de participantes con dificultades o no para reunirse y construir un consenso grupal (García-Valdés \& Suárez-Marín, 2013).

La validación de contenido de la escala permitió concluir que esta logra evaluar los factores de riesgo de maltrato del adulto mayor con claridad en la redacción, coherencia interna en cada ítem, sin inducción a las respuestas o sesgo, con un lenguaje adecuado a la población, dejando solo los ítems relevantes y permitiendo que estos fueran suficientes para la evaluación de las tres dimensiones propuestas, de manera que se ha obtenido un criterio de validez sobre el contenido de la escala, aunque como lo propone Pedrosa, et al. (2013) no es suficiente y requiere de la aplicación de otros procesos que permitan dar cuenta de otros tipos de validez y fiabilidad de la escala. 
La escala sometida al juicio de expertos estaba conformada por 91 ítems y dividida en 12 dimensiones, tomando como base el trabajo realizado por Giraldo-Rodríguez (2006); sin embargo, la versión aprobada por los jueces expertos queda conformada por 80 ítems y tres dimensiones que representan tres factores de riesgo coincidentes con la clasificación compilada por Pabón y Delgado (2017), estos son, los factores de riesgo asociados al adulto mayor, factores de riesgo asociados al cuidador, factores de riesgo asociados al entorno, las instituciones y la cultura; dicha versión se obtiene a través de tres procesos de evaluación con cuatro jueces expertos, que realizaron la evaluación tres veces, tres jueces en cada ocasión hasta obtener el total acuerdo entre los mismos, tal como lo plantea el método de agregados individuales (Corral, 2009).

En cuanto al reporte de maltrato, este estudio encontró que la mayoría de evaluados fueron víctimas en el último año $(66,7 \%)$, principalmente de maltrato psicológico, aunque también es común que se dé más de un tipo de maltrato, lo que es coincidente con el reporte en otros estudios como el realizado en siete países europeos por Eslami et al. (2016), destacando una prevalencia de una vida de maltrato psicológico de 35,17 \% y otros estudios colombianos que reflejan una prevalencia importante de maltrato psicológico, como los de Cardona-Arango et al. (2010) con una prevalencia de 15,8 \%, García y Medina (2009), y Sepúlveda et al. (2009). Así mismo, se encontró que el agresor, generalmente era el esposo(a) o nuero(a), aunque también se dio un alto reporte que indica que el maltrato se produjo por varios familiares, contrario a lo evidenciado por Ruelas-González et al. (2016) quienes evidencian que en la mayoría de los casos el agresor no tiene un vínculo filial.

Respecto a los factores de riesgo, se logra evidenciar que de los asociados a los gerontes representan mayor riesgo los factores económicos, ya que hay cuatro ítems altamente puntuados en este factor; Bover et al. (2003) y Centro Nacional de Excelencia Tecnológica en Salud (2013) apoyan lo hallado cuando mencionan la falta o insuficiencia de recursos financieros como riesgo económico, y en la muestra incluida en este estudio, se agrega el depender económicamente de la familia, ganar menos de un mínimo, estar económicamente inactivo, y no recibir ningún tipo de pensión.

Por su lado, los factores físicos y cognitivos presentan solo dos ítems que indican riesgo y finalmente, el factor emocional ninguno, lo que resulta contrario a los reportes actuales en el tema; Moreno y González (2010) 
indican como riesgo un estado de salud deficiente, deterioro cognitivo y aislamiento social, en la misma línea Tabueña (2009) incluye el estar enfermo, dependencia física y emocional; la muestra estudiada reporta una buena percepción de salud (75 \%) y percepción de baja dificultad para realizar las actividades de la vida diaria $(33,3 \%)$, a pesar de que se destaca la presencia de alteraciones visuales ( $83 \%$ ) y presencia de dolores intensos en diferentes partes del cuerpo (54,2\%), presentar olvidos frecuentes (75\%), dificultad para concentrarse y alteraciones del sueño (50,5\%); los evaluados no sienten que esto les afecte su desenvolvimiento cotidiano.

En cuanto a los factores emocionales los mayores reportan que no sienten miedo $(79,2 \%)$, no se sienten mal al tratar con los demás $(91,7 \%)$ y sienten que tienen una persona de confianza a la que le pueden comunicar sus cosas $(87,5 \%)$, lo que no resulta coincidente con lo propuesto por Moreno y González (2010), quienes resaltan dentro de los factores emocionales la baja autoestima; lo anterior, se puede relacionar con el hecho de que los seniles se encuentran integrados a grupos de autoayuda o centros vida, espacios que significan un apoyo social importante para los mismos y permiten un impacto sobre su calidad de vida contrarrestando los factores de riesgo; para Fernández-Ballesteros et al. (2010) y Martín-Lesende et al. (2007) la vinculación y participación social favorecen un envejecimiento saludable con la reducción de la presencia de enfermedades físicas y malestar emocional.

Finalmente, del riesgo asociado a los mayores, los evaluados indican en el riesgo por negligencia que $50 \%$ de la muestra prefiere no pedir ayuda aun cuando la necesiten y optan estar callados y no molestar a nadie (75 \%), pero suelen seguir instrucciones médicas y acudir a controles (75\%), lo que no muestra un resultado contundente de autonegligencia, pero representa una variable que valdría la pena profundizar, ya que la baja capacidad de autocuidado incrementa la probabilidad de maltrato (Adams, 2012; Bover et al., 2003) y deteriora la calidad de vida del mayor (Fernández-Ballesteros et al., 2010).

Respecto al riesgo asociado a los cuidadores, Bover et al. (2003), Iborra (2008), Moreno y González (2010), y Tabueña (2009) destacan sobrecarga física, ser hijo o pareja de la víctima, asumir otras obligaciones además de la de cuidar al mayor; los resultados obtenidos en este estudio refieren que $90,5 \%$ de estos pertenecen a la propia familia, ninguno se dedica de forma exclusiva al cuidado del mayor, dedicando tiempo a otras funciones $(57,1 \%)$, y no reciben pago (100\%); el $30 \%$ de los seniles perciben que su cuidador 
se altera con facilidad, aunque ninguno consume alcohol, y pocos consumen medicamentos psiquiátricos (5\%), refiriendo poco riesgo según las características del cuidador, contrario a lo que reportan autores como Pabón, Galvis y Cerquera (2014), quienes hacen énfasis en las consecuencias negativas que se desencadenan por asumir el rol de cuidador, y lo que agregan Pabón y Delgado (2017), quienes indican que la carga emocional y física de los cuidadores incrementan el riesgo de maltrato; sin embargo, este resultado es coincidente con su percepción adecuada de salud física y nivel de independencia.

En relación a los factores de riesgo asociados al entorno, la cultura y las instituciones se encontró que 52,2 \% refiere que la vejez representa solo pérdidas, dato a destacar, ya que la percepción que se tenga del envejecimiento y la vejez van a repercutir de forma importante sobre el tipo de envejecimiento y la probabilidad de sufrir maltrato (Cerquera, Álvarez \& Saavedra, 2010; Fernández-Ballesteros et al., 2010); 47,8 \% afirma que durante su infancia sufrió maltrato por parte de sus padres, lo que se enmarca en la teoría explicativa del maltrato denominada aprendizaje social, descrita por Adams (2012) como la tendencia de los individuos a replicar en la edad adulta las conductas de maltrato aprendidas durante la infancia. Así, se encuentran solo dos elementos de riesgo de maltrato asociados a este factor, aunque para Iborra (2008), Moreno y González (2010), y Tabueña (2009) también podrían serlo la pobreza y escasa preparación educativa.

Lo anterior, permite dar cuenta de pocos factores de riesgo, los mayores en general son independientes y activos, las funciones de apoyo brindadas por los familiares son pocas, aunque se destacan estereotipos negativos sobre la vejez por parte de los mismos gerontes y limitaciones económicas; el reporte realizado es netamente descriptivo, por lo que se recomienda complementar la descripción que se puede obtener con otros instrumentos y variables asociadas al fenómeno del maltrato y establecer relaciones; el riesgo de maltrato es un tema sobre el que es necesario profundizar, según Moreno y González (2010), la identificación de los factores de riesgo es esencial para poder actuar antes de que el abuso esté consumado.

En cuanto a la prueba piloto, se logra concluir que la mejor forma de aplicar la escala es de forma administrada e individualmente, donde dicha aplicación puede tener una duración entre 15 y 30 minutos; se evidencia una correcta comprensión de los ítems por parte de los seniles y a menos que no se use un tono de voz adecuado (especialmente si el mayor presenta alteraciones 
auditivas) no surgen preguntas o dudas por los términos utilizados en la formulación de los ítems.

Se recomienda para trabajos posteriores incrementar la muestra de estudio, incluir análisis de datos más complejos, que permitan un proceso psicométrico de alcance correlacional o multivariado, estableciendo la relación entre la presencia de maltrato y los factores de riesgo incluidos; a pesar de que los factores de riesgo propuestos en la escala se han analizado por medio de diferentes estudios en el exterior e incluso uno en Colombia demostrando la relación entre sí; es importante establecer un grupo normativo que sirva de referente para determinar los factores de riesgo de maltrato en población mayor, estos finalmente servirán de apoyo para que el profesional de psicología direccione una intervención adecuada.

Financiación: Proyecto financiado por la Universidad de Investigación y Desarrollo UDI Seccional Bucaramanga: Proyecto Validación por jueces expertos de la Encuesta sobre maltrato a personas adultas mayores en el Distrito Federal EMPAM-DF con acto administrativo 2016-006-1A.

Agradecimientos: A cada una de las personas mayores que con disposición permitieron a la investigadora indagar y acceder a su información personal para aportar al estudio del maltrato y contribuir a la psicogerontología

\section{REFERENCIAS}

Adams, Y. (2012). Maltrato en el adulto mayor institucionalizado. Revista Médica clínica las Condes, 23(1), 84-90. https://doi.org/10.1016/S0716-8640(12)70277-8

Aragón, L. (2011). Evaluación Psicológica: historia, fundamentos teórico-conceptuales y psicometría. México: Manual Moderno.

Ato, I., López, J., \& Benavente, A. (2013). Un sistema de clasificación de los diseños de investigación en psicología. Anales de Psicología, 29(3), 1038-1059. http://dx.doi. org/10.6018/analesps.29.3.178511

Barrón, M., Sánchez, A., \& Méndez, G. (2002). Factores que influyen en el maltrato al adulto mayor. Revista Especializada en Ciencias de la Salud, 5(1-2), 21-24. http:// www.revistas.unam.mx/index.php/vertientes/article/view/33231/30415

Bover, A.A., Moreno, M.L., Mota, S., \& Taltavull, L.M. (2003). El maltrato a los ancianos en el domicilio. Situación actual y posibles estrategias de intervención. Atención primaria, 32(9), 541-551. https://doi.org/10.1016/S0212-6567(03)70785-9

Cano, S., Garzón, M., Segura, A. \& Cardona, D. (2015). Factores asociados al maltrato del adulto mayor de Antioquia. Revista Facultad Nacional de Salud Pública, 33(1), 67-74. http://aprendeenlinea.udea.edu.co/revistas/index.php/fnsp/article/view /18825/18024

Cardona-Arango, D., Estrada-Restrepo, A., Chavarriaga-Maya, L., Segura-Cardona, A., Ordóñez-Molina, J., \& Osorio-Gómez, J. (2010). Apoyo social dignificante del adulto mayor institucionalizado. Medellín, 2008. Revista de Salud Pública, 12(3), 
414-424. Recuperado de: http://www.bdigital.unal.edu.co/16118/1/1095975832-1-PB.pdf

Centro Nacional de Excelencia Tecnológica en Salud (2013). Guía de práctica clínica: Detección y manejo del maltrato en el adulto mayor. Cuauhtémoc: CENETEC. http://www.cenetec.salud.gob.mx/descargas/gpc/CatalogoMaestro/057_GPC_ MaltratoAdultoMayor/MAM_EVR_CENETEC.pdf

Cerquera-Córdoba, A. M., Álvarez-Agudelo, J.L., \& Saavedra-Ruiz, A.C. (2010). Identificación de estereotipos y prejuicios hacia la vejez presentes en una comunidad educativa de Florida Blanca. Psychologia. Avances de la disciplina, 4(1), 73-87. http://www.redalyc.org/articulo.oa?id=297224086007

Corral, Y. (2009). Validez y confiabilidad de los instrumentos de investigación para la recolección de datos. Revista Ciencias de la Educación, 19(2), 228-247. http:// servicio.bc.uc.edu.ve/educacion/revista/n33/art12.pdf

Departamento Administrativo Nacional de Estadística (DANE, 2009). Proyecciones de población 2005-2020. Recuperado de http://www.dane.gov.co/files/investigaciones/poblacion/proyepobla06_20/8Tablasvida1985_2020.pdf

Escobar-Pérez, J., \& Cuervo-Martínez, T. (2008). Validez de contenido y juicio de expertos: una aproximación a su utilidad. Avances en medición, 6, 27-36. http:// www.humanas.unal.edu.co/psicometria/files/7113/8574/5708/Articulo3_ Juicio_de_expertos_27-36.pdf

Eslami, B., Viitasara, E., Macassa, G., Melchiorre, M., Lindert, J., Stankunas, M., \& Soares, J. (2016). The prevalence of lifetime abuse among older adults in seven European countries. International Public Health, 61, 891-901. https://10.1007/ s00038-016-0816-x

Fernández, M. (2015). Malos tratos a las personas mayores: una realidad creciente y oculta. Formación Médica Continuada en Atención Primaria, 22(7), 357-359. https://10.1016/j.fmc.2015.03.021

Fernández-Ballesteros, R., Zamarrón, M.D., López, M.D., Molina, M.A., Díez, J., Montero, P., \& Schettini, R. (2010). Envejecimiento con éxito: criterios y predictores. Psicothema, 22(4), 641-647. http://www. psicothema.com/pdf/3779.pdf

García, Z., \& Medina, P. (2009). Caracterización del maltrato al adulto mayor, denunciado en los años 2006 y 2007 en las comisarías de familia de las localidades de Usaquén y Rafael Uribe Uribe de la ciudad de Bogotá D.C. (Tesis pregrado). Universidad de San Buenaventura, Colombia. http://biblioteca.usbbog.edu. co:8080/Biblioteca/BDigital/44522.pdf

García Valdés, M., \& Suárez Marín, M. (2013). El método Delphi para la consulta a expertos en la investigación científica. Revista Cubana de Salud Pública, 39(2) 253-267. http://scielo.sld.cu/scielo.php?script=sci_arttext\&pid=S0864-34662013000200007

Giraldo, L. (2012). El maltrato de las personas de edad. En: M. Gutiérrez-Robledo y J. Kershenobich-Stalnikowitz (Eds.), Envejecimiento y salud: una propuesta para un plan de acción. México: Academia Nacional de Medicina de México, Academia Mexicana de Cirugía A.C., Instituto de Geriatría y Universidad Nacional Autónoma de México. http://envejecimiento.sociales.unam.mx/archivos/GERIATRIAenvysaludweb.pdf

Giraldo Rodríguez, M. (2006). Análisis de la información estadística. Encuesta sobre maltrato a personas adultas mayores en el distrito federal. La Esperanza: Gobierno del Distrito Federal. http://www.sideso.cdmx.gob.mx/documentos/ analisi_estadistico_sobre_maltrato_a_adultos_mayores.pdf 
Giraldo-Rodríguez, M. \& Rosas-Carrasco, C. (2013). Development and psychometric properties of the Geriatric Mistreatment Scale. Geriatrics and gerontology International, 12, 466-474. https://10.1111/j.1447-0594.2012.00894.x

Huenchuan, S. (2012). Los derechos de las personas mayores en el siglo XXI: situación, experiencias y desafíos. En: M.A. Mautone (Eds), Igualdad y no discriminación por edad. México D.F: CEPAL. https://repositorio.cepal.org/bitstream/ handle/11362/1465/S301435D4312012_es.pdf?sequence=1\&isAllowed=y

Iborra, I. (2008). Maltrato de las personas mayores en la familia en España. Valencia: Fundación de la Comunitat Valenciana para el estudio de la violencia (Centro Reina Sofía). Recuperado de http://inger.gob.mx/bibliotecageriatria/acervo/ pdf/58iborramaltrato.pdf

Loaiza-Valencia, A. (2015). Situación demográfica y epidemiológica de los adultos mayores. Santander. 1994-2011. Revista MedUNAB, 18(1), 58-65. Recuperado de http://revistas.unab.edu.co/index.php?journal=medunab\&page=article\&op=view\&path\%5B\%5D=2161

Martín- Lesende, I., Martín-Zurro, A., Moliner-Prada, C. \& Aguilera-Gracía, L. (2007). Envejecimiento activo, la mejor receta para prevenir la dependencia. Revista Española de Geriatría y Gerontología, 42(2), 4-6. https://doi.org/10.1016/ S0211-139X(07)75733-5

Mora-Ríos, J., Bautista-Aguilar, N., Natera, G., \& Pedersen, D. (2013). Adaptación cultural de instrumentos de medida sobre estigma y enfermedad mental en la ciudad de México. Salud Mental, 36(1), 9-18. http://www.scielo.org.mx/pdf/sm/ v36n1/v36n1a2.pdf

Moreno, S., \& González, M. (2010). Malos tratos a las personas mayores: una realidad oculta. Recuperado de: http://envejecimiento.csic.es/documentos/documentos/ caixacatalunya-malostratos-01.pdf

Pabón, D.K., \& Delgado, J.A. (2017). Maltrato en la población adulta mayor: una revisión. Espacio Abierto: Cuaderno Venezolano de Sociología, 26(2), 245-267. http://produccioncientificaluz.org/index.php/espacio/article/view/22819/22706

Pabón, D.K., Galvis, M.A., \& Cerquera, A.M. (2014). Diferencias en el apoyo social percibido entre cuidadores informales y formales de pacientes con demencia tipo Alzheimer. Psicología desde el Caribe, 31(1), 59-77. http://www.redalyc.org/ articulo. oa?id=21330429004.

Pedrosa, I., Suárez-Álvarez, J. \& García-Cueto, E. (2013). Evidencias sobre la validez de contenido: avances teóricos y métodos para su estimación. Acción Psicológica, 10(2), 3-18. https://doi.org/10.5944/ap.10.2.11820

Rodríguez, K. (2011). Vejez y envejecimiento. Documento de Investigación. Escuela Médicos y Ciencias de la Salud, 12, 1-42. http://www.urosario.edu.co/urosario_ files/dd/dd857fc5-5a01-4355-b07a-e2f0720b216b.pdf

Ruelas-González, M., Duarte-Gómez, M., Flores-Hernández, S., Ortega-Altamirano, D., Cortés-Gil, J., Taboada, A. \& Ruano, L. (2016). Prevalence and factors associated with violence and abuse of older adults in Mexico's 2012 National Health and Nutrition Survey. International Journal for Equity in Health, 15(31). https://10.1186/s12939-016-0315-y

Sepúlveda Carrillo, G., Arias, J., Cuervo, A., Gutiérrez, S., Oliveros, S \& Valbuena, A. (2009). Caracterización de los casos de maltrato en el adulto mayor denunciados en la comisaría de familia en la localidad de Usaquén en el año 2007. Revista Colombiana de Enfermería, 4(4), 1-10. http://revistacolombianadeenfermeria. unbosque.edu.co/article/view/1411/1017. 
Tabueña, M. (2009). Los malos tratos a las personas mayores, una realidad oculta. Obras sociales de Caixa Catalunya. Recuperado de http://envejecimiento.csic.es/ documentos/documentos/caixacatalunya-malostratos-01.pdf

Touza, C., Prado, C., \& Segura, M.P. (2011). Construcción y validación de las escalas de detección de riesgo de malos tratos domésticos y comportamientos autonegligentes (EDMA). International Journal of Psychology and Psychological Therapy, 11(1), 91-106. http://www.ijpsy.com/volumen11/num1/284/construccin-y-validacin-de-las-escalas-ES.pdf.

Urrutia, M., Barrios, S., Gutiérrez, M., \& Mayorga, M. (2014). Métodos óptimos para determinar validez de contenido. Educación Superior, 28(3), 547-558. http:// scielo.sld.cu/pdf/ems/v28n3/ems14314.pdf autor o el licenciante.

\section{(cc) BY}




\section{Anexo 1. Formato evaluación por parte del experto}

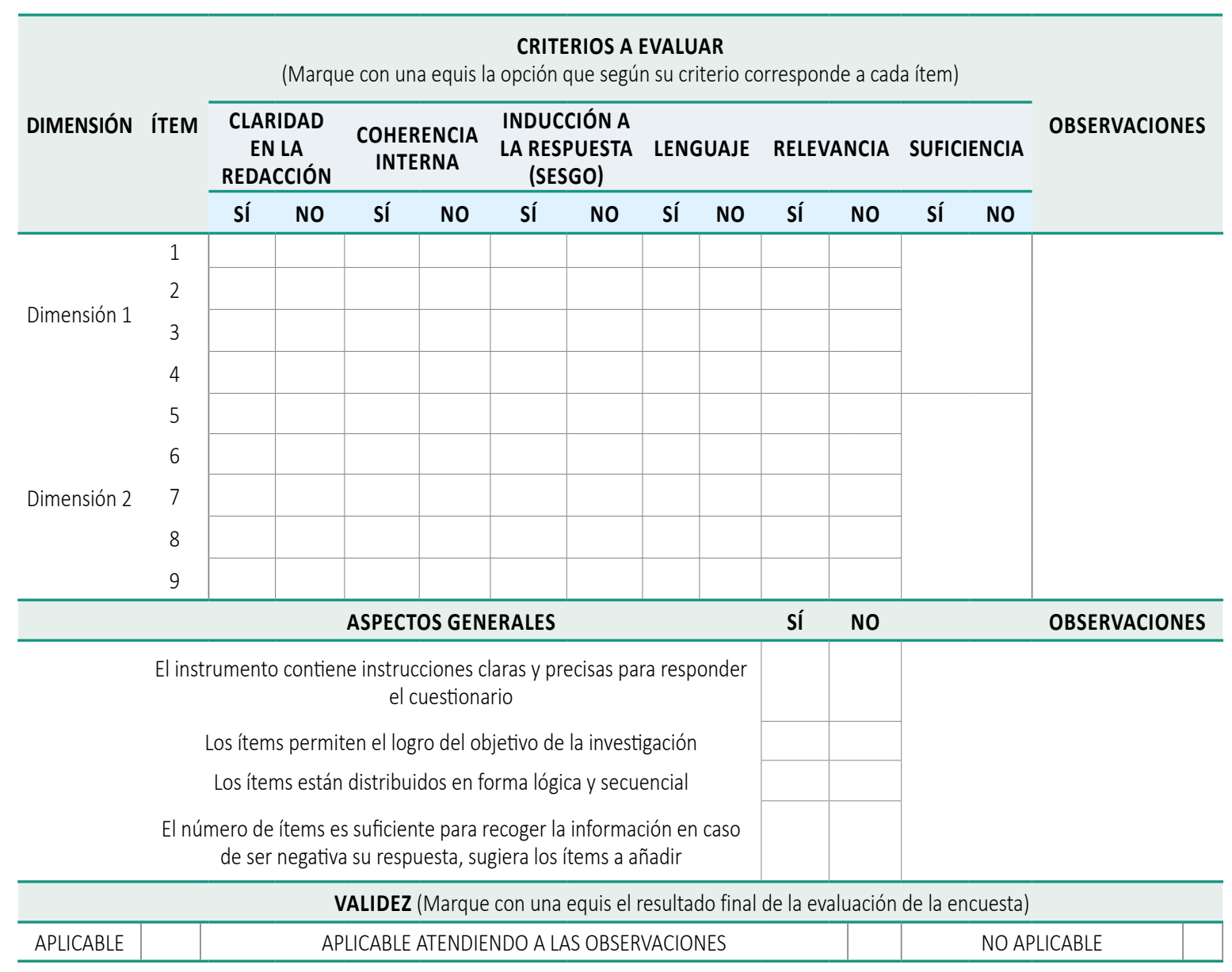

Fuente: Propia autoría formato adaptado de: Corral, Y. (2009) y Escobar-Pérez, J. (2008)

\section{Anexo 2. ENCUESTA SOBRE RIESGO DE MALTRATO EN PERSONAS MAYORES}

La siguiente encuesta debe ser aplicada no autoadministrada. Diligencia los siguientes datos:

\begin{tabular}{l}
\hline FECHA: \\
\hline Nombre: \\
Dirección: \\
Sexo: \\
Edad:
\end{tabular}


Escolaridad:

Estrato:

Vive con: (número de personas)

Nombre cuidador o persona de apoyo: (si tiene)

Parentesco:

Diagnósticos médicos (físico, psicológico, psiquiá-

trico, neurológico, neuropsicológico)

IMC:

Leer la instrucción textualmente al entrevistado: "A continuación le voy a leer algunas afirmaciones. Usted me va a responder sí o no, según corresponda a su caso, recuerde que ninguna de las opciones es correcta o incorrecta y tenga en cuenta su experiencia en los últimos seis meses para responder"

\section{FACTORES DE RIESGO ADULTO MAYOR}

Físicos

Presenta alteraciones visuales

Las alteraciones visuales le dificultan realizar las actividades diarias

Presenta alteraciones auditivas

Las alteraciones auditivas le dificultan realizar las actividades diarias

Está diagnosticado con alguna patología crónica

La(s) enfermedades crónicas le generan dependencia

Sufre de dolores físicos intensos

Usted percibe su salud como deficiente

\section{Emocionales}

Siente que tiene una persona de confianza a la que le puede comunicar sus problemas

Ha sentido pérdida de interés por las cosas que generalmente le gusta hacer

Se siente asustado(a) o con miedo.

Llora con facilidad

Se siente a menudo aburrido

Se irrita con facilidad

Últimamente se ha sentido deprimido(a)

últimamente se ha sentido solo(a)

Siente miedo de alguien

Tiene miedo de que le suceda algo malo

Se siente desconfiado o tímido

Se siente mal al tratar de relacionarse con los demás

Ha sentido deseos de irse a otro lugar porque se siente mal en donde vive y con quien vive 


\section{Cognitivos}

Ha presentado olvidos frecuentes

Ha presentado problemas por los olvidos que presenta

Se desorienta con facilidad perdiendo noción del tiempo (fecha/hora)

Se desorienta con facilidad perdiendo la ubicación del lugar en el que se encuentra

Se le dificulta concentrarse

Sufre de alteraciones del sueño

Ha sufrido pérdida de la conciencia por caída o golpe en la cabeza

\section{Económicos}

\begin{tabular}{|c|c|c|}
\hline & sí & NO \\
\hline \multicolumn{3}{|l|}{ Tiene ingresos mensuales propios } \\
\hline \multicolumn{3}{|l|}{ Sus ingresos son superiores al mínimo legal vigente $(\$ 678.400)$} \\
\hline \multicolumn{3}{|l|}{ Depende económicamente de su familia } \\
\hline \multicolumn{3}{|l|}{ Recibe pensión } \\
\hline \multicolumn{3}{|l|}{ Está jubilado } \\
\hline \multicolumn{3}{|l|}{ Actualmente trabaja } \\
\hline \multicolumn{3}{|l|}{ Trabaja, pero no es remunerado } \\
\hline \multicolumn{3}{|l|}{ Actualmente se dedica a quehaceres dentro del hogar } \\
\hline \multicolumn{3}{|l|}{ Necesita de la ayuda de alguien para manejar su dinero } \\
\hline \multicolumn{3}{|l|}{ Puede disponer de su dinero y hacer gastos con libertad } \\
\hline Delega en otra persona el manejo de su dinero & & \\
\hline
\end{tabular}

\section{Autonegligencia}

Descuida su cuidado personal

Prefiere no pedir ayuda aun si la necesita

Incumple las instrucciones o recomendaciones médicas

Prefiere no acudir al médico aun cuando lo requiere

No asiste a los controles médicos programados

Prefiere estar callado(a) y no molestar a nadie

\section{FACTORES DE RIESGO ASOCIADAS AL CUIDADOR}

Cuenta con una persona de apoyo que lo cuida cuando lo requiere

La persona que lo apoya en sus actividades pertenece a su familia

La persona que lo apoya se dedica exclusivamente a ello

La persona que lo apoya le dedica tiempo a otras funciones

La persona que lo cuida recibe pago por ello

Mantiene una comunicación positiva con la persona que lo apoya

Percibe una relación positiva con la persona que lo apoya en sus actividades diarias

La persona que lo apoya depende económicamente de usted 
La persona de apoyo se adapta a las condiciones para poder darle cuidados

La persona que apoya su cuidado consume alcohol con frecuencia

La persona que lo apoya se altera con facilidad

La persona que lo apoya consume medicamentos psiquiátricos

\section{FACTORES DE RIESGO ASOCIADOS AL ENTORNO, LA CULTURA Y LAS} INSTITUCIONES

La casa en donde vive es de su propiedad

Usted paga arriendo

Usted tiene su propia habitación

Cuenta con servicios públicos básicos

Su lugar de vivienda es aislado

Tiene acceso a servicios de salud

La zona en donde vive es insegura

Se ha visto afectado por la inseguridad de la zona en donde vive

Ha recibido algún tipo de maltrato en instituciones, empresas, entidades, locales por ser usted

$$
\text { una persona mayor }
$$

Se ha sentido maltratado por algún profesional de la salud

Se ha sentido atendido por personal poco capacitado

Tiene una relación cercana con sus hijos

Tiene una relación cercana con sus nietos

Siente que en su familia piensan que por ser mayor es una carga

Usted piensa que por ser mayor es una carga

Piensa que la vejez presenta solo pérdidas

En su familia hablan de forma negativa de las personas mayores

En su familia han existido casos de maltrato

Alguna vez se ha denunciado un caso de maltrato en su familia

En su barrio conoce casos de maltrato

En su infancia sufrió maltrato por parte de sus padres

En su matrimonio sufrió maltrato por parte de su pareja

Siente que le ofrecen servicios deficientes por ser mayor 